\title{
Measuring rabbit (Oryctolagus cuniculus) tooth growth and eruption by fluorescence markers and bur marks
}

\author{
Wyss, Fabia ; Müller, Jacqueline ; Clauss, Marcus ; Kircher, Patrick R ; Geyer, Hans ; von Rechenberg, Brigitte ; \\ Hatt, Jean-Michel
}

\begin{abstract}
Rabbits (Oryctolagus cuniculus) and rodents possess continuously growing teeth, and dental problems are a major health issue in these species. Knowledge of tooth growth characteristics is required to adequately treat dental problems and advise owners concerning diets. Most research was performed using bur marks and measuring eruption and wear manually. However, this method cannot be applied to teeth less rostral than the first premolar; therefore, for evaluation of molars, other methods are needed. We evaluated the use of fluorochromes xylenol orange and calcein green to measure growth rates of rabbit teeth and compared this method to results obtained by manually measuring the distance between a bur mark and the gingival margin of the same tooth (eruption) and by measuring the distance between the bur mark and the apex of the same tooth on computed tomography scans (growth). Apical fluorochrome measurements correlated well with eruption and growth rates obtained with bur marks, whereas measurements coronal to the pulp cavity did not. Growth rates were approximately $1.9 \mathrm{~mm} / \mathrm{wk}$ for maxillary and $2.2 \mathrm{~mm} / \mathrm{wk}$ for mandibular incisors. Growth rates of premolars were $2.14+0.28 \mathrm{~mm} / \mathrm{wk}$ in rabbits on a grass/rice hulls/sand pelleted diet and $0.93+0.18 \mathrm{~mm} / \mathrm{wk}$ in rabbits on a hay diet. Growth of molars could only be assessed using the measurement in dentin on the wall of the pulp cavity, which does not account for the real growth. However, being similar to this measurement in premolars, one could hypothesize similar growth in molars as in premolars. We conclude that the application of fluorochrome staining can be used to measure tooth growth in teeth that are not accessible for bur marks or in animals that are too small to assess tooth eruption or growth by bur marks.
\end{abstract}

DOI: https://doi.org/10.1177/0898756416640956

Posted at the Zurich Open Repository and Archive, University of Zurich

ZORA URL: https://doi.org/10.5167/uzh-124372

Journal Article

Published Version

Originally published at:

Wyss, Fabia; Müller, Jacqueline; Clauss, Marcus; Kircher, Patrick R; Geyer, Hans; von Rechenberg, Brigitte; Hatt, Jean-Michel (2016). Measuring rabbit (Oryctolagus cuniculus) tooth growth and eruption by fluorescence markers and bur marks. Journal of Veterinary Dentistry, 33(1):39-46.

DOI: https://doi.org/10.1177/0898756416640956 


\title{
Measuring Rabbit (Oryctolagus cuniculus) Tooth Growth and Eruption by Fluorescence Markers and Bur Marks
}

Journal of Veterinary Dentistry 2016, Vol. 33(I) 39-46 (C) The Author(s) 2016 Reprints and permission: sagepub.com/journalsPermissions.nav DOI: $10.1177 / 0898756416640956$ jov.sagepub.com (S)AGE

\author{
Fabia Wyss, Dr. med. vet.', Jacqueline Müller, Dr. med. vet.', \\ Marcus Clauss, Prof. Dr. med. vet., MSc, Dipl. ECVCN', \\ Patrick Kircher, Prof. Dr. med. vet., PhD, Dipl. ECVDI', \\ Hans Geyer, Prof. em. Dr. med. vet. ${ }^{3}$, \\ Brigitte von Rechenberg, Prof. Dr. med. vet., Dipl. ECVS ${ }^{4}$, and \\ Jean-Michel Hatt, Prof. Dr. med. vet., MSc, Dipl. ACZM, Dipl. ECZM (avian)'
}

\begin{abstract}
Rabbits (Oryctolagus cuniculus) and rodents possess continuously growing teeth, and dental problems are a major health issue in these species. Knowledge of tooth growth characteristics is required to adequately treat dental problems and advise owners concerning diets. Most research was performed using bur marks and measuring eruption and wear manually. However, this method cannot be applied to teeth less rostral than the first premolar; therefore, for evaluation of molars, other methods are needed. We evaluated the use of fluorochromes xylenol orange and calcein green to measure growth rates of rabbit teeth and compared this method to results obtained by manually measuring the distance between a bur mark and the gingival margin of the same tooth (eruption) and by measuring the distance between the bur mark and the apex of the same tooth on computed tomography scans (growth). Apical fluorochrome measurements correlated well with eruption and growth rates obtained with bur marks, whereas measurements coronal to the pulp cavity did not. Growth rates were approximately $1.9 \mathrm{~mm} / \mathrm{wk}$ for maxillary and $2.2 \mathrm{~mm} / \mathrm{wk}$ for mandibular incisors. Growth rates of premolars were $2.14 \pm 0.28 \mathrm{~mm} / \mathrm{wk}$ in rabbits on a grass/rice hulls/sand pelleted diet and $0.93 \pm 0.18 \mathrm{~mm} / \mathrm{wk}$ in rabbits on a hay diet. Growth of molars could only be assessed using the measurement in dentin on the wall of the pulp cavity, which does not account for the real growth. However, being similar to this measurement in premolars, one could hypothesize similar growth in molars as in premolars. We conclude that the application of fluorochrome staining can be used to measure tooth growth in teeth that are not accessible for bur marks or in animals that are too small to assess tooth eruption or growth by bur marks.
\end{abstract}

\section{Keywords}

lagomorph, tooth, wear, growth, measurement, incisor, molar, diet

\section{Introduction}

Lagomorphs and rodents possess long-crowned (hypsodont), continuously growing (elodont) and open-rooted (aradicular) teeth. ${ }^{1,2}$ In lagomorphs and hystricomorph rodents, both incisor and cheek teeth show these characteristics, unlike other rodents that have closed-rooted (brachydont) premolars and molars. ${ }^{1,3}$ This continuous growth is not constant but compensates for the wear of the respective tooth, ${ }^{4,5}$ and it was shown that dentin apposition on the incisor teeth is reduced in squirrels during hibernation-a situation with no wear. ${ }^{6}$

Measuring tooth growth in lagomorphs and rodents was mostly performed by the use of bur marks on incisor teeth, ${ }^{4,5,7,8}$ and this is also possible in the premolars of larger species. ${ }^{4}$ In doing so, the change in the distance between the bur mark and the gingival border - that is, the eruption rate - is used as a proxy for growth. Eruption rate and growth rate are not necessarily the same; various studies of rabbits and rodents have shown that for a real assessment of growth, not only the visible tooth crown, but also the complete tooth has to be measured. ${ }^{9-11}$

\footnotetext{
' Vetsuisse Faculty, Clinic for Zoo Animals, Exotic Pets and Wildlife, University of Zurich, Zurich, Switzerland

${ }^{2}$ Vetsuisse Faculty, Division of Diagnostic Imaging, University of Zurich, Zurich, Switzerland

${ }^{3}$ Vetsuisse Faculty, Institute of Veterinary Anatomy, University of Zurich, Zurich, Switzerland

${ }^{4}$ Vetsuisse Faculty, Center for Applied Biotechnology and Molecular Medicine (CABMM) and Musculoskeletal Research Unit (MRSU), Equine Hospital, University of Zurich, Zurich, Switzerland
}

\section{Corresponding Author:}

Marcus Clauss, Vetsuisse Faculty, Clinic for Zoo Animals, Exotic Pets and Wildlife, University of Zurich, Winterthurerstr. 260, 8057 Zurich, Switzerland. Email: mclauss@vetclinics.uzh.ch 
Because tooth growth and eruption are not only different between incisors and cheek teeth, ${ }^{4}$ but may also vary among cheek teeth (premolars and molars) according to their position, a method to monitor the growth or eruption of the not easily accessible premolars and molars would be a valuable additional tool. The use of fluorescent markers that binds to growing bone and tooth tissue administered to the animals at a known time interval, and measurement of the distance between the thus-marked growth lines, has been described. ${ }^{12}$ Over 50 years ago, Alizarin Red was injected as a vital dye to produce sharp red lines in dentin and bone calcifying at the time $^{13}$ and was also used to evaluate open-rooted teeth in squirrels. ${ }^{6}$ Tetracycline markers were also used to stain bone and teeth and determine growth in humans, dogs, and rats. ${ }^{14,15}$ A novel technique to enhance bone and dental labeling using spectral decomposition and subsequent linear unmixing was developed to depict each individual fluorochrome without interference of any other. ${ }^{16}$ Seven fluorochromes were successfully tested-calcein blue, xylenol orange, calcein, alizarin complexone, doxycycline/rolitetracycline, hematoporphyrin, and 1,2-bis(2-aminophenoxy) ethane- $N, N, N^{\prime}, N^{\prime}$-tetraacetic acid-for their use to determine bone growth. ${ }^{16,17}$ All these fluorochromes were shown later to accumulate in dentin as well so that they can be used to evaluate tooth growth. ${ }^{17}$

Being large enough for a range of examination techniques (macroscopic inspection, computed tomography [CT]) and due to their easy maintenance, rabbits (Oryctolagus cuniculus) represent attractive animal models to evaluate tooth growth and wear. ${ }^{18-21}$ This study is part of a larger experimental study on tooth wear, eruption, and growth of rabbits fed different diets. ${ }^{4}$ The aim of this study was to evaluate the correlation of manual measurements using bur marks (eruption) with measurements on CT scans (growth) and measurements obtained from fluorescence microscopic images of the rabbit's teeth after administration of 2 fluorochrome markers (growth).

\section{Methods}

During an experiment on the influence of dietary abrasives on wear and growth in rabbits, ${ }^{4}$ we compared 3 potential methods of measuring tooth growth: manual measurements in manually restrained animals, measurements from CT images as well as measurements obtained from fluorescence microscopic images of the teeth. This study was performed during the last 20 days of the above-mentioned experiment.

\section{Animals and Diets}

The experiment was approved by the Cantonal Veterinary Office in Zurich, Switzerland (No. 80/2012). Six female New Zealand White rabbits were selected for this study during the end phase of the other study. Because animals were aged approximately 8.5 months at this time, a major influence of body growth on eruption measurements due to changes in gingival tissue or alveolar bone was not expected (but not assessed). All rabbits were kept individually in hutches $(1.00 \times 0.75 \mathrm{~m})$ on wood shavings and with plastic hides without other gnawing opportunities except their diet. Water was provided ad libitum. Three rabbits each were transferred to a diet of grass hay $(\mathrm{H})$ or grass and rice hull pellets with an addition of sand (GRS) ${ }^{4}$ fed ad libitum. Two to 5 days later and 21 days prior to euthanasia, $5 \mathrm{mg} / \mathrm{kg}$ body weight of the fluorescence marker calcein green ${ }^{\mathrm{a}}$ was injected subcutaneously. Ten days later ( \pm 1 hour), subcutaneous injection of 90 $\mathrm{mg} / \mathrm{kg}$ body weight of the second fluorescence marker xylenol orange ${ }^{b}$ was performed.

\section{Dental Nomenclature}

The dental nomenclature used in this study corresponds to the previous study ${ }^{4}$ to facilitate comparison: For maxillary and mandibular incisors, $\mathrm{I}^{1}$ and $\mathrm{I}_{1}$ were used, respectively, and mandibular (lower) cheek teeth were denoted by lower case letters ( $\mathrm{p}$ or $\mathrm{m}$ ). Phylogenetic positions were represented by numbering of the cheek teeth (ie, p3 and p4, m1-m3). ${ }^{2}$ Maxillary cheek teeth were not used in this study.

\section{Bur Mark Measurements}

Manual measurements of tooth eruption were performed as previously described ${ }^{4}$ using bur marks on incisors and $\mathrm{p} 3$ by measuring the distance between the gingival margin and the mark (Manual) — on the labial (incisor) and the mesial (p3) surface, respectively. Computed tomographic images as also previously described ${ }^{4}$ were used to measure the distance from the apex of the respective tooth to the bur mark, representing growth. Bur marks were identified on the CT images, and the distance from the apex to the closer edge of the bur mark was measured using a polygon of 5 to 7 data points, depending on the curvature (CT; Figure 1). These measures were again performed on the labial (incisor) and the mesial (p3) surface, respectively. Tooth growth was calculated as the difference of the apex-bur mark-length between 2 time points.

\section{Fluorescence Marker Measurements}

After euthanasia, the right side of the mandible and the maxillary incisors were separated from soft tissue and were stored in $50 \%$ ethanol for 245 days before processing. The mandibular bone was separated using an electrical drill machine ${ }^{c}$ with a diamond cutting $\operatorname{disc}^{\mathrm{d}}$ to obtain flat cut surfaces of the incisors (longitudinal and craniocaudal) and of the cheek teeth p4 and m1 (both longitudinal and mediolateral). All samples were processed using established protocols. ${ }^{22}$ They were first dehydrated in increasing grades of ethanol ${ }^{\mathrm{e}}$, then bathed in xylene $^{\mathrm{f}}$ as an intermedium and in methyl methacrylate ${ }^{\mathrm{g}}$, followed by block-embedding in methyl methacrylate mixture (89.5\% methyl methacrylate, $10 \%$ dibutyl phthalate, ${ }^{\mathrm{h}}$ and $0.5 \%$ Perkadox $16^{\mathrm{i}}$ over 6 days). Sections of $700 \mu \mathrm{m}$ were cut using a diatome blade $\mathrm{saw}^{\mathrm{j}}$ and then glued $^{\mathrm{k}}$ on commercial histology slides. 


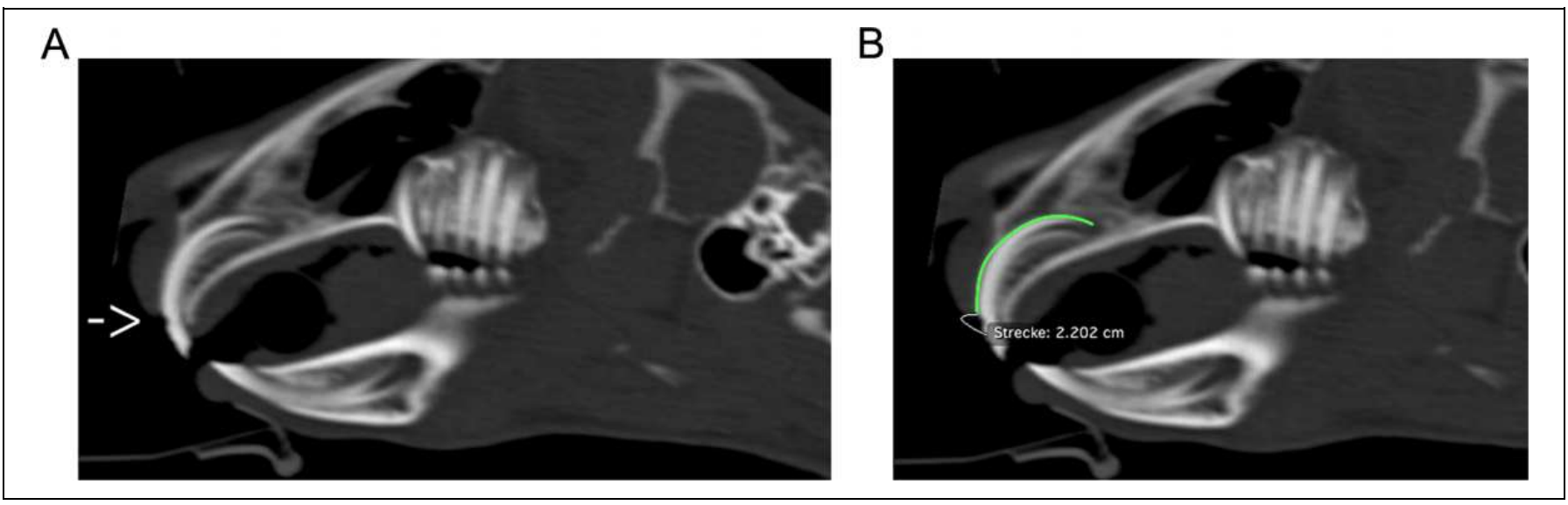

Figure I. Sagittal reconstruction of a computed tomographic image from a rabbit showing the bur mark on the labial side of the maxillary incisor $(A ;$ arrow) and the polygon with 5 data points to measure the distance between apex and bur mark (B).

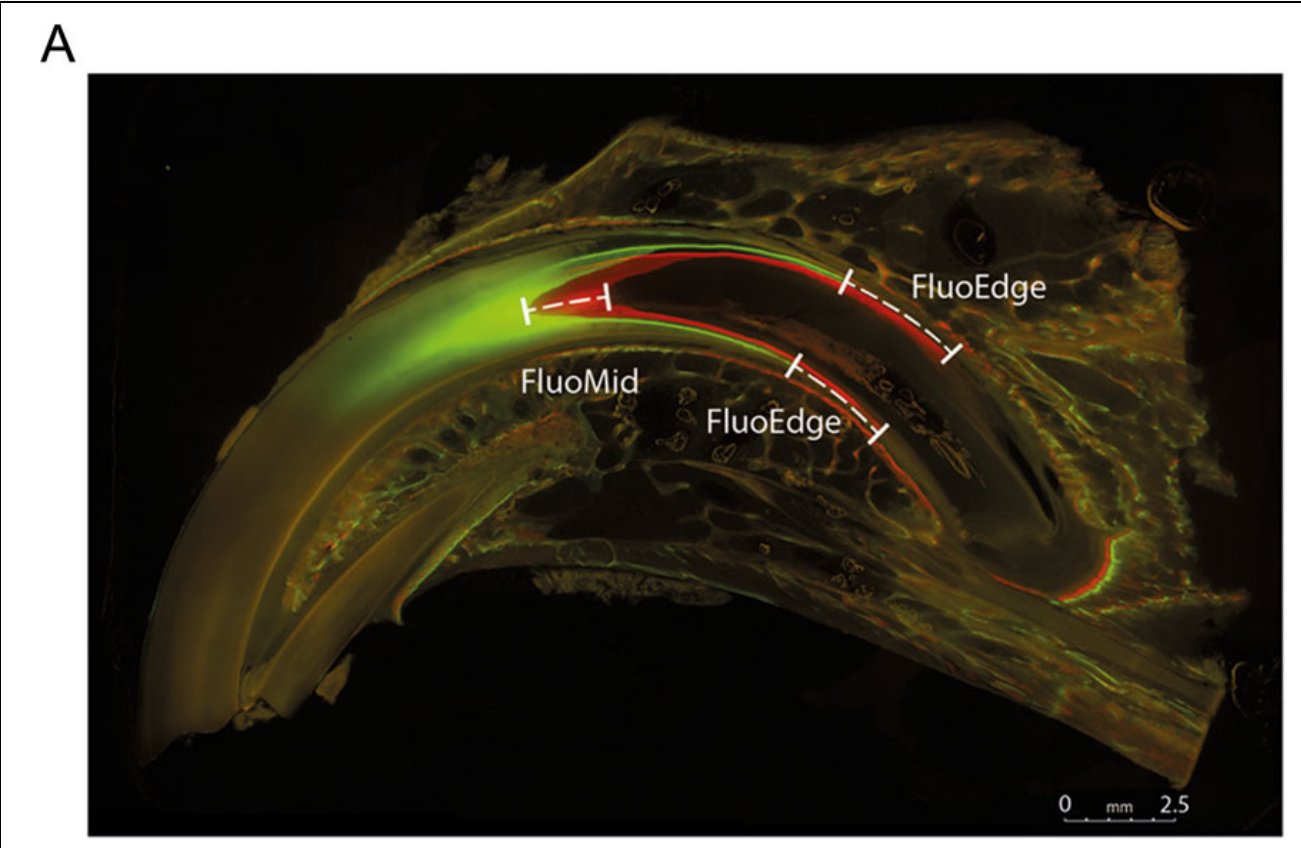

\section{B}

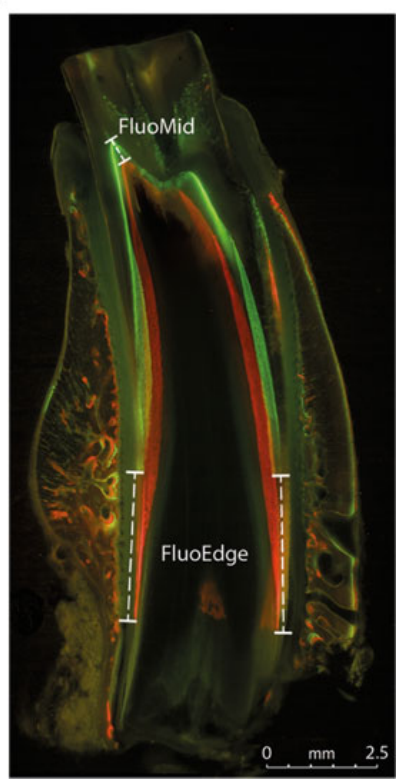

Figure 2. Fluorescence microscopic image of a rabbit's maxillary incisor (A) and a mandibular premolar (B) tooth. Fluorescence microscopic marks of calcein green and xylenol orange are delineated to measure the distance between the 2 marks at 2 different locations: apically on the labial or buccal, respectively, and lingual dentin surfaces (FluoEdge) and coronally from the tip of the pulp cavity within the dentin (FluoMid).

Fluorescence images were taken in TIF format using a fluorescence microscope (Leica DM 6000B, Leica Microsystems, Heerbrugg, Switzerland, Leica CTR 6000, Leica Microsystems, Heerbrugg, Switzerland) with the according software (LAS AF 6000, Leica Microsystems, Heerbrugg, Switzerland). Two special filters were used (L5 for calcein green, N3 for xylenol orange) and were combined to an overlay image. Measurements of distances between the 2 fluorescence marks were then performed using the free download software Image J. ${ }^{1}$ Measurements were performed on different locations of the teeth for incisors on the labial and lingual surfaces and for cheek teeth on the buccal and lingual surfaces, respectively (FluoEdge; values represent averages of both measures for each tooth). Additionally, measurements were taken, in both incisors and cheek teeth, coronally from the tip of the pulp cavity of the reserve crown, within the tooth (FluoMid; Figure 2).

\section{Statistics}

Fluorescence marker measurements of the same tooth were compared using paired $t$ tests. Correlations between measurements of the different methods were assessed, due to the low sample size, by nonparametric (Spearman's $\rho$ ) correlation. Finally, we performed a general linear model (GLM) using FluoEdge as the dependent variable, Manual as the independent variable, and tooth, diet, and the 3 interactions Diet $\times$ Tooth, Diet $\times$ Manual, and Tooth $\times$ Manual as covariables, with a stepwise removal of the term with the highest $P$ value 
Table I. Dental Growth and Eruption Rates (mean \pm SD, in mm per week) in the Rabbit as Measured by 3 Different Methods. ${ }^{\text {a }}$

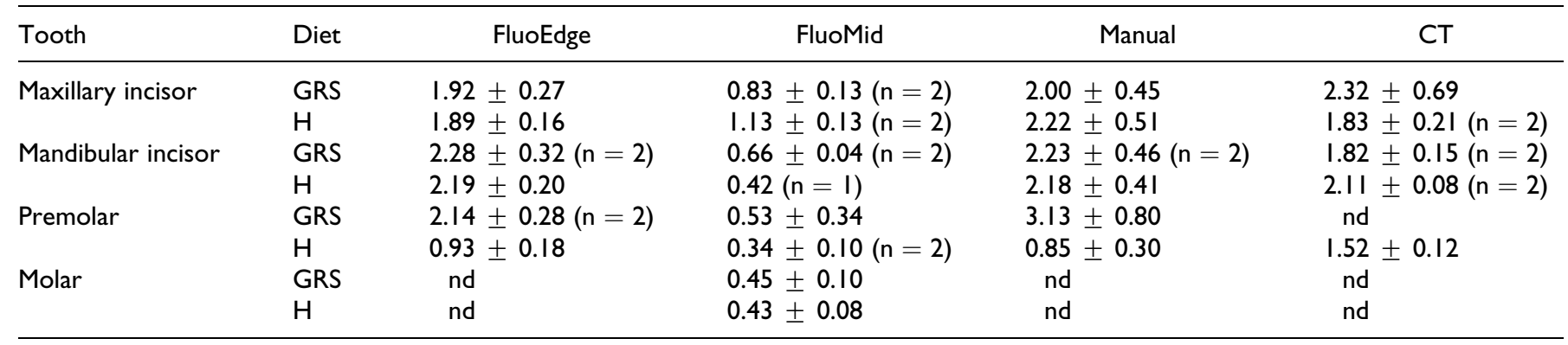

${ }^{a}$ Distances on fluorescence microscopic images of the teeth, manual measurements, and CT measurements; $\mathrm{n}=3$ unless otherwise noted.

Abbreviations: CT, computed tomography; FluoEdge, apically on the outer surface of dentin; FluoMid, measurement coronally from the tip of the pulp cavity, within the dentin (cf Figure I); GRS, grass and rice hull pellets with an addition of sand; H, grass hay; nd, measurements were not possible; SD, standard deviation.

until only significant terms were retained (and confirming normal distribution of the residuals of the final model). Analyses were performed in SPSS 21.0 (SPSS Inc, Chicago, Illinois), with the significance level set to .05 .

\section{Results}

Almost all examined teeth could be assessed using the 3 different measurement methods-manual, CT, and fluorescence microscopy. However, bur marks on the premolars in rabbits receiving diet GRS were already worn off when the next CT scan was performed and therefore, neither manual nor CT measurements could be made in these teeth (indicated by "nd" in Table 1). Furthermore, fluorescence microscopic marks on the outer surface on all 3 examined molar teeth were not as clearly identifiable as would have needed to be able to measure the distance between the 2 marks (also indicated by "nd" in Table 1).

Distances between the 2 fluorescence microscopic measures from the 2 different dental locations (FluoEdge vs FluoMid; Table 1; Figure 1) revealed large differences between locations (paired $t$ tests for $\mathrm{I}^{1} P=.007, \mathrm{I}_{1} P=.017$, p3 $P=$ .047 ) without a significant correlation (Table 2; Figure 3A). FluoMid also did not correlate with Manual or CT measurements (Table 2; Figure 3B and C). However, when comparing FluoEdge with the Manual measurement of the same animals, a significant correlation was observed (Table 2; Figure 3D). A similar correlation was found when comparing these fluorescence measurements with CT measurements (Table 2; Figure 3E). Manual and CT measurements also showed a significant correlation (Table 2; Figure 3F). Growth estimated by FluoEdge of maxillary and mandibular incisors as well as premolars was around $2 \mathrm{~mm} / \mathrm{wk}$ (Table 1). FluoMid in molars resembled that of premolars (Table 1). Our sample size was too small to assess differences between diets, although there was an apparent difference in tooth eruption and growth of the premolars, with higher growth on the sand diet as reflected in Manual and FluoEdge measurements, which corresponds to previous findings of this study. ${ }^{4}$

The final GLM using FluoEdge as the dependent variable and Manual as the independent variable had a high adjusted
Table 2. Interactions Between Dental Growth and Eruption Rates in the Rabbit Obtained by 3 Different Measurements (Manual, Computed Tomography, and Fluorescence Microscopic) and 2 Different Locations Measured on Fluorescence Microscopy Images.

\begin{tabular}{|c|c|c|c|c|}
\hline Tooth & Measure & CT & FluoEdge & FluoMid \\
\hline Maxillary incisor & $\begin{array}{l}\text { CT } \\
\text { FluoEdge }\end{array}$ & $\begin{array}{l}\mathbf{n}=\mathbf{5} \\
\rho=.900 \\
\boldsymbol{P}=\mathbf{. 0 3 7}\end{array}$ & $\begin{array}{l}\mathbf{n}=\mathbf{6} \\
\rho=.943 \\
\mathbf{P}=.005 \\
\mathbf{n}=\mathbf{5} \\
\rho=.900 \\
\mathbf{P}=.037\end{array}$ & $\begin{array}{l}n=4 \\
\rho=.400 \\
P=.600 \\
n=4 \\
\rho=.400 \\
P=.600 \\
n=4 \\
\rho=.400 \\
P=.600\end{array}$ \\
\hline Mandibular incisor & FluoEdge & $\begin{array}{l}n=4 \\
\rho=.00 \\
P=1.0\end{array}$ & $\begin{array}{l}\mathbf{n}=\mathbf{5} \\
\rho=.900 \\
\mathbf{P}=.037 \\
\mathrm{n}=4 \\
\rho=.00 \\
P=1.0\end{array}$ & $\begin{array}{l}\mathrm{n}=3 \\
\rho=.500 \\
P=.667 \\
\mathrm{n}=3 \\
\rho=-1.0 \\
P<.001 \\
\mathrm{n}=3 \\
\rho=.500 \\
P=.667\end{array}$ \\
\hline Premolar p3 & $\mathrm{CT}$ & $\begin{array}{l}n=3 \\
\rho=.500 \\
P=.667\end{array}$ & $\begin{array}{l}\mathbf{n}=\mathbf{5} \\
\rho=.900 \\
\boldsymbol{P}=.037 \\
\mathbf{n}=\mathbf{3} \\
\rho=1.0 \\
\boldsymbol{P}<.001\end{array}$ & $\begin{array}{l}\mathrm{n}=5 \\
\rho=.100 \\
\mathrm{P}=.873 \\
\mathrm{n}=2 \\
\quad \text { na }\end{array}$ \\
\hline & FluoEdge & & & $\begin{array}{l}\mathrm{n}=4 \\
\rho=.400 \\
P=.600\end{array}$ \\
\hline All teeth & CT & $\begin{array}{l}\mathbf{n}=\mathbf{I 2} \\
\rho=.734 \\
\boldsymbol{P}=.007\end{array}$ & $\begin{array}{l}\mathbf{n}=\mathbf{I 6} \\
\rho=.803 \\
\boldsymbol{P}<.001 \\
\mathbf{n}=12 \\
\rho=.741 \\
\boldsymbol{P}=.006\end{array}$ & $\begin{array}{l}\mathrm{n}=12 \\
\rho=.147 \\
P=.649 \\
\mathrm{n}=9 \\
\rho=.300 \\
P=.433 \\
\mathrm{n}=11 \\
\rho=.064 \\
P=.853\end{array}$ \\
\hline
\end{tabular}

Abbreviations: CT, computed tomography; FluoEdge, apically on the outer surface of dentin; FluoMid, measurement coronally from the tip of the pulp cavity, within the dentin; na, not analyzed. Significant meaningful correlations set in bold. 


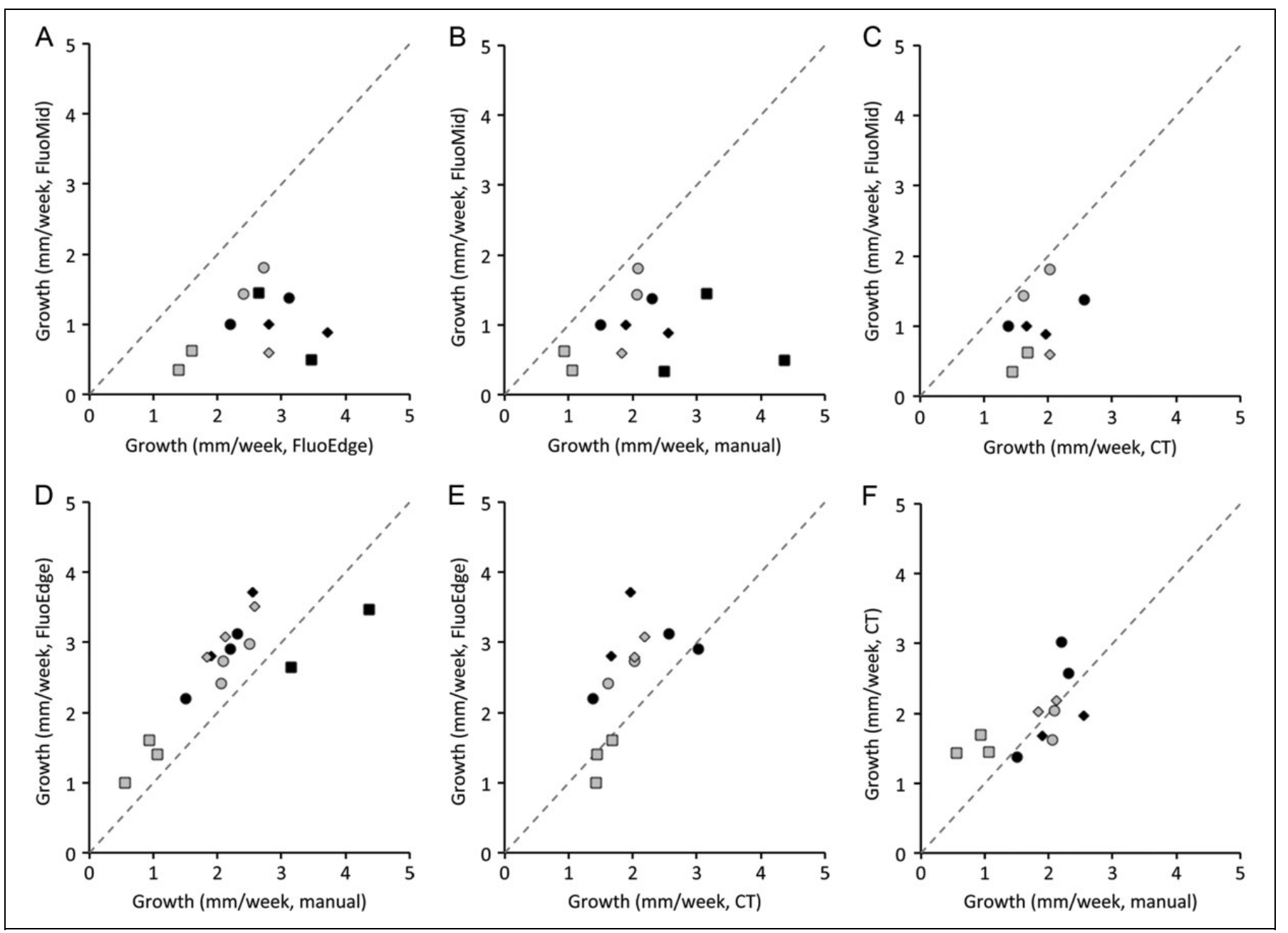

Figure 3. Correlations between tooth growth measurements of distances between 2 fluorescence microscopic marks on 2 different locations (FluoEdge and FluoMid), tooth eruption rate obtained by manual and tooth growth rate obtained by computed tomographic (CT) measurements in the rabbit (in mm per week; circles indicate maxillary incisors; diamonds, mandibular incisors; squares, premolars; black, sand diet; grey, hay diet; the dotted line represents $y=x$ ). A, Poor correlation of distances between the 2 fluorescence microscopic marks (FluoEdge and FluoMid). B, Poor correlation between manually measured eruption rates and FluoMid. C, Poor correlation between growth rates on CT images and FluoMid. D, Good correlation between manually measured eruption rates and FluoEdge. E, Moderate-to-good correlation between measurements on CT images and FluoEdge. F, Moderate-to-good correlation between measurements on CT images and manually measured eruption rates.

$R^{2}$ of 0.96, with a significant intercept $\left(F_{1,12}=59.776, P<\right.$ $.001)$, a significant effect of Manual $\left(F_{1,12}=369.696, P<\right.$ $.001)$, and a significant Tooth $\times$ Manual interaction $\left(F_{2,12}\right.$ $=70.332, P<.001$ ), which corresponds to the visual impression of Figure 3D, where the deviation of the 2 premolar measurements on the sand diet account for the significant interaction effect.

\section{Discussion}

Fluorochromes such as the ones used in this study (calcein green, xylenol orange) have been used in bone and teeth and tested for their usefulness in determining growth. ${ }^{16,17}$ The good correlation of the distance between fluorescence microscopic markers (FluoEdge) and the eruption rate measurements obtained by manual measurements ${ }^{4}$ and growth measurements taken from CT scans indicate that the fluorescence microscopic marker method is appropriate to evaluate growth rates in rabbit teeth and could potentially also be used to measure growth rates of teeth that are not accessible to bur marks. The correlation between eruption rate (Manual) and growth rate (CT measurements) suggests that conditions leading to a systematic difference between these 2 measures, such as difference in occlusion between individual teeth or an effect of overall body growth, were of limited influence in this study. However, the fact that both measures did not match completely could have been caused by the fact that the period that the animals received a constant diet in this experiment 
was too short to result in a complete physiological equilibrium. Not only tooth eruption and growth but also tooth wear and eruption may either only achieve complete (static) equilibrium after a longer period on a constant diet than in our experiment ${ }^{4,5}$; alternatively, findings of fluctuating wear and eruption even during long periods of consistent diets ${ }^{23}$ could indicate that a real steady state may be impossible to achieve. In free-ranging animals and animals feeding on natural forages, constancy of wear, that is, dietary abrasiveness and amounts ingested (and hence chewed), cannot be expected. Therefore, wear, eruption, and growth might best be conceptualized as part of a fluctuating equilibrium.

When applying fluorescence microscopic markers to evaluate growth rates, some considerations of caution need to be taken into account.

Welfare considerations. The use of fluorescence microscopic markers evidently requires the sacrifice of experimental animals. Their application therefore should be restricted to investigations for which the other measures cannot be applied, such as the molar growth rates. Because the fluorescence microscopic markers were not reported to cause toxicities at the dosages used ${ }^{24,25}$ and are excreted from vascularized tissue within 2 hours, ${ }^{25}$ the use of experimental animals, after slaughtering, as food for other animals is feasible and safe. The dose of xylenol used in our study was similar to the recommend dose in rabbits $(90 \mathrm{mg} / \mathrm{kg})$, whereas we only used half or a quarter of the recommended dose $(5 \mathrm{mg} / \mathrm{kg}$ instead of $10 \mathrm{mg} / \mathrm{kg}$ or $20 \mathrm{mg} /$ $\mathrm{kg}$, respectively) of calcein green to label bone tissue. ${ }^{24,26}$ Nevertheless, the lower dose used in our study stained the growth line in the teeth very well, and we can recommend this lower dose for the use of teeth staining in rabbits.

Positioning of teeth during histological preparation. The distance between 2 fluorescent growth marks will be at its maximum when the plane of the histological section is exactly parallel to the direction of growth. In practice, it is unlikely that this is achieved, even if the positioning of the teeth during cutting is performed with great care. Therefore, measures taken from fluorescence microscopic markers should be considered estimates. In the present study, finding the correct position for cutting the molars so that FluoEdge measurements were possible was not achieved, highlighting the difficulty of performing such sections.

Location of measurement within teeth. Measurements of distance between 2 fluorescence microscopic marks differed significantly between the 2 locations measured in this study (FluoEdge and FluoMid). This is in accordance to previous findings, ${ }^{12}$ where measurements were most accurate at the dentinoenamel border. Measurement of FluoMid thus does not account for the real growth and would lead to underestimation of the growth rate. The odontoblasts that are responsible for the growth measured as FluoMid produce the secondary dentin most probably in an oblique direction and at a lower rate. Additionally, the mentioned problem of a histological section that is not perfectly parallel to the direction of growth will affect the measurement. Nevertheless, FluoMid may be used to estimate the growth rate of the molars, from which the distance of the 2 fluorescence microscopic marks could not be assessed on the outer surface of the tooth. Being similar to FluoMid measured in premolars, it could be hypothesized that growth rates in premolars and molars are similar.

Growth rates measured in this study (FluoEdge) were approximately $1.9 \mathrm{~mm} / \mathrm{wk}$ for maxillary incisors (1.92 \pm $0.27 \mathrm{~mm} / \mathrm{wk}$ and $1.89 \pm 0.16 \mathrm{~mm} / \mathrm{wk}$, for GRS diet and $\mathrm{H}$ diet, respectively), $2.2 \mathrm{~mm} / \mathrm{wk}$ for mandibular incisors (2.28 \pm $0.32 \mathrm{~mm} / \mathrm{w}$ and $2.19 \pm 0.20 \mathrm{~mm} / \mathrm{wk}$, for GRS diet and $\mathrm{H}$ diet, respectively). This is slightly lower or similar to incisor growth rates mentioned in the literature, ${ }^{12,21,27}$ although diet may play an important role ${ }^{4,8}$ but was not taken into account in most publications.

Premolars grew $2.14 \pm 0.28 \mathrm{~mm} / \mathrm{wk}$ in rabbits on GRS diet and $0.93 \pm 0.18 \mathrm{~mm} / \mathrm{wk}$ in rabbits on $\mathrm{H}$ diet. The difference in growth rate between the 2 diets was not assessed in the present study due to the small sample size, but it is in accordance with wear and eruption measurements in a higher number of animals using bur marks. ${ }^{4}$ Most authors suspected growth rates of cheek teeth to be lower than $1 \mathrm{~mm} / \mathrm{wk}$ and especially lower than incisor growth rates, ${ }^{21,28-30}$ except for 1 study based on enamel staining ${ }^{12}$ that documented growth rates in rabbits of more than $1 \mathrm{~mm} / \mathrm{wk}$. In our own study, growth rates of premolars and possibly molars were similar to those of incisors in rabbits fed diet GRS but not on diet H, indicating that growth of different teeth adjusts to the specific situation triggered by a specific diet. ${ }^{4,9}$

In conclusion, the present study demonstrates that fluorescent markers can be conveniently used to determine growth rates in rabbit teeth that are comparable with those measured by other methods. The results document growth rates of premolars and molars that are higher than previously suspected and that respond flexibly with respect to the actual abrasion induced by the ingested diet.

\section{Materials}

a. Calein green, $1 \mathrm{~mL} / \mathrm{kg} 0.5 \%$ solution, calcein disodium salt; Sigma Aldrich GmbH, Buchs, Switzerland.

b. Xylenol orange, $1 \mathrm{~mL} / \mathrm{kg} 9 \%$ solution, xylenol orange tetrasodium salt; Sigma Aldrich $\mathrm{GmbH}$, Buchs, Switzerland.

c. Dremel 395; Bosch AG, Otelfingen, Switzerland.

d. Proxxon No 28 840; Proxxon GmbH, Föhren, Germany.

e. $50 \%$ to $100 \%$ ethanol; Thommen Furler AG, Rüti b. Büren, Switzerland.

f. Xylene; Thommen Furler AG, Rüti b. Büren, Switzerland.

g. Methyl methacrylate; Sigma Aldrich GmbH, Buchs, Switzerland.

h. Dibutylphtalate; Sigma Aldrich GmbH, Buchs, Switzerland. 
i. Perkadox 16; Dr. Grogg, Chemie AG, Stettlen, Switzerland.

j. Diatome blade saw, Exakt Trennschleifsystem $300 \mathrm{CP}$; Exakt Apparatebau GmbH, Norderstedt, Germany.

k. Cementit CA12, Merz + Benteli AG, Niederwangen, Switzerland.

1. ImageJ, Rasband, W.S., ImageJ; US National Institutes of Health, Bethesda, Maryland, http://imagej.nih.gov/ij/, 1997-2014.

\section{Acknowledgments}

The authors thank Katharina Kämpf, Ladina Ettinger Ferguson, and Aymone Lenisa for their assistance in processing the samples, and Dr Karina Klein for assistance in taking microscopic images. Two anonymous reviewers contributed greatly to the correctness of the manuscript.

\section{Declaration of Conflicting Interests}

The author(s) declared no potential conflicts of interest with respect to the research, authorship, and/or publication of this article.

\section{Funding}

The author(s) received no financial support for the research, authorship, and/or publication of this article.

\section{References}

1. Vella D, Donnelly TM. Rabbits: Basic anatomy, physiology, and husbandry. In: Quesenberry KE, Carpenter JW, eds. Ferrets, Rabbits, and Rodents: Clinical Medicine and Surgery. 3rd ed. St. Louis, MO: Elsevier; 2012:157-173.

2. Ungar PS. Mammal Teeth: Origin, Evolution and Diversity. Baltimore, MD: John Hopkins University Press; 2010.

3. Lennox AM, Bauck L. Small rodents: Basic anatomy, physiology, husbandry, and clinical techniques. In: Quesenberry KE, Carpenter JW, eds. Ferrets, Rabbits, and Rodents: Clinical Medicine and Surgery. 3rd ed. St. Louis, MO: Elsevier; 2012: 339-353.

4. Müller J, Clauss M, Codron D, et al. Growth and wear of incisor and cheek teeth in domestic rabbits (Oryctolagus cuniculus) fed diets of different abrasiveness. J Exp Zool A. 2014;321(5):283-298.

5. Müller J, Clauss M, Codron D, et al. Tooth length and incisal wear and growth in guinea pigs (Cavia porcellus) fed diets of different abrasiveness. J Anim Physiol Anim Nutr. 2015;99(3):591-604.

6. Sarnat BG, Hook WE. Effects of hibernation on tooth development. Anatomical Record 1942;83(4):471-493.

7. Shadle AR. The attrition and extrusive growth of the four major incisor teeth of domestic rabbits. J Mammol. 1936;17(1):15-21.

8. Wolf P, Kamphues J. Probleme der art- und bedarfsgerechten Ernährung kleiner Nager als Heimtiere. Der praktische Tierarzt. 1995;88(12):1088-1092.

9. Ness AR. The response of the rabbit mandibular incisor to experimental shortening and to the prevention of its eruption. Proc $R$ Soc Lond B Biol Sci. 1956;146(922):129-154.
10. Taylor AC, Butcher EO. The regulation of eruption rate in the incisor teeth of the white rat. J Exp Zool. 1951;117(1): 165-188.

11. Risnes S, Septier D, Goldberg M. Accelerated eruption of rat lower incisor. Relationship between impeded and unimpeded eruption rates, rate of attrition, tooth length, and production of dentin and enamel. Connect Tissue Res. 1995;32(1-4): 183-189.

12. von Koenigswald W, Golenishev FN. A method for determining growth rates in continuously growing molars. J Mammol. 1979; 60(2):397-400.

13. Schour I, Hoffman MM, Sarnat BG, Engel NB. Vital staining of bones and teeth with Alizarine Red S. J Dent Res. 1941;20(20):411-418.

14. Lee WR. Appositional bone formation in canine bone: a quantitative microscopic study using tetracycline markers. J Anat. 1964; 98(4):665-677.

15. Harris WH. A microscopic method of determining rates of bone growth. Nature. 1960;188(4755):1038-1039.

16. Pautke C, Vogt S, Tischer T, et al. Polychrome labeling of bone with seven different fluorochromes: enhancing fluorochrome discrimination by spectral image analysis. Bone. 2005;37(4): 441-445.

17. Pautke $\mathrm{C}$, Tischer $\mathrm{T}$, Vogt $\mathrm{S}$, et al. New advances in fluorochrome sequential labelling of teeth using seven different fluorochromes and spectral image analysis. J Anat. 2007;210(1): 117-121.

18. Capello V, Cauduro A. Clinical technique: application of computed tomography for diagnosis of dental disease in the rabbit, guinea pig, and chinchilla. J Exotic Pet Med. 2008;17(2): 93-101.

19. Van Caelenberg AI, De Rycke LM, Hermans K, Verhaert L, van Bree HJ, Gielen IM. Computed tomography and cross-sectional anatomy of the head in healthy rabbits. Am J Vet Res. 2010;71(3): 293-303.

20. Van Caelenberg AI, De Rycke LM, Verhaert L, Van Bree HJ, Gielen IM. Comparison of radiography and CT to identify changes in the skull of four rabbits with dental disease. $J$ Vet Dent. 2011;28(3):172-181.

21. Jekl V, Redrobe S. Rabbit dental disease and calcium metabolism - the science behind divided opinions. J Small Anim Pract. 2013;54(9):481-490.

22. Leutenegger CM, von Rechenberg B, Huder JB, et al. Quantitative real-time PCR for equine cytokine mRNA in nondecalcified bone tissue embedded in methylmethacrylate. Calcif Tissue Int. 1999;65(5):378-383.

23. Wolf P, Kamphues J. Untersuchungen zu Fütterungseinflüssen auf die Entwicklung der Incisivi bei Kaninchen, Chinchilla und Ratte. Kleintierpraxis. 1996;41(10):723-732.

24. Olerud S, Lorenzi GL. Triple fluorochrome labeling in bone formation and bone resorption. J Bone Joint Surg. 1970;52(2): 274-278.

25. Rahn BA, Perren SM. Xylenol orange, a fluorochrome useful in polychrome sequential labeling of calcifying tissues. Biotech Histochem. 1971;46(3):125-129. 
26. von Gaalen SM, Kruyt MC, Geuze RE, de Bruijn JD, Alblas J, Dhert WJ. Use of fluorochrome labels in in vivo bone tissue engineering research. Tissue Eng Part B Rev. 2010;16(2): 209-217.

27. Harcourt-Brown F.Dental disease in pet rabbits 1 . Normal dentition, pathogenesis and aetiology. In Practice. 2009;31(8): 370-379.
28. Schumacher M. Measurement of clinical crown length of incisor and premolar teeth in clinically healthy rabbits. J Vet Dent. 2011; 28(2):90-95.

29. Meredith A. Rabbit dentistry. Eur J Companion Anim Pract. 2007; 17(1):55-62.

30. Lord B. Dental disease in the rabbit Part 1: normal dentition and diet. Companion Anim. 2011;16(5):53-55. 